\title{
Distributed and Power Efficient Routing in Wireless Cooperative Networks
}

\author{
Zhengguo Sheng*, Zhiguo Ding †, and Kin K Leung* \\ *Department of Electrical and Electronic Engineering, Imperial College, UK \\ $\dagger$ Department of Communication Systems, Lancaster University, UK \\ Email: zhengguo.sheng06@imperial.ac.uk
}

\begin{abstract}
Most ad hoc mobile devices in wireless networks operate on batteries and power consumption is therefore an important issue for wireless network design. In this paper, we propose and investigate a new distributed cooperative routing algorithm that realizes minimum power transmission for each composed cooperative link, given the link BER (Bit Error Rate) constrained at a certain target level. The key contribution of the proposed scheme is to bring the performance gain of cooperative diversity from the physical layer up to the networking layer. Specifically, the proposed algorithm selects the best relays with minimum power consumption in distributed manner, and then forms cooperative links for establishing a route with appropriate error performance from a source to a destination node. Analytical results are developed to show that our cooperative transmission strategy (MPSDF) achieves average energy saving of $82.43 \%$ compared to direct transmission, and of $21.22 \%$ compared to the existing minimum power cooperation strategy. Furthermore, the proposed power efficient routing algorithm can also reduce the total power consumption by a couple $\mathrm{dB}$ compared to existing cooperative routing algorithms. Monte-Carlo simulation results are also provided for performance evaluation.
\end{abstract}

\section{INTRODUCTION}

As one of the most important technologies nowadays, wireless sensor network plays a more and more important role in vast applications, such as military communication and disaster or infrastructures monitoring. The deployment of the low battery cost and size limited sensors implies that energy efficient communication protocol is essential to extend the network lifetime [1], [2].

Meanwhile, as a new research area, cooperative transmission has attracted much attention as an effective technique to combat multi-path fading, enhance receiver reliability and achieve better energy efficiency of wireless communication systems [3], [4]. The problem of energy saving can be approached from physical layer, MAC layer up to application layer. Moreover, the cross layer approach has been shown to be an effective way in the energy efficient routing design. Various energy concerned cooperative routing algorithms have been developed at the physical layer to further reduce the

This research was sponsored by US Army Research laboratory and the UK Ministry of Defence and was accomplished under Agreement Number W911NF-06-3-0001. The views and conclusions contained in this document are those of the authors and should not be interpreted as representing the official policies, either expressed or implied, of the US Army Research Laboratory, the U.S. Government, the UK Ministry of Defense, or the UK Government. The US and UK Governments are authorized to reproduce and distribute reprints for Government purposes notwithstanding any copyright notation hereon.

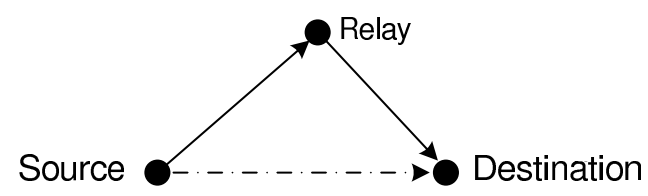

Fig. 1. An example of a one-hop wireless cooperative link

total transmission power of cooperative transmission [5]-[9]. However, most of existing cooperative routing algorithms are implemented by identifying a shortest path first and thus the performance gains of cooperative communication cannot be fully exploited. As a contrary, our proposed routing algorithm starts with routes with a small number of hops and forms the relationship between the minimum power of cooperative transmission and its BER performance.

To be specific, we first propose a new distributed cooperative transmission strategy, namely, Minimum Power Selection Decode-and-Forward (MPSDF), which realizes minimum power cooperative transmission, given the link BER constrained at a certain target level. This new method combines the physical and MAC layer mechanisms to identify the best relay in a cooperative and distributed way. Furthermore, combining with MPSDF, the proposed power efficient routing algorithm can identify a minimum power route while guaranteeing the desired QoS.

The reminder of this paper is organized as follows. In Section II, we define the system model of cooperative network and derive a closed form expression for MPSDF. In Section III, we describe our distributed and power efficient routing algorithm. In Section IV, we show the numerical results for our proposed scheme and paper concludes in Section V.

\section{SySTEM MODEL}

In cooperative networks, each node acts two roles in the network transmission: source node and relay node. Here, relay transmission is a main feature of cooperative communication. Figure 1 shows a cooperative network model.

A cooperative link (CL) between the source and destination nodes includes two different transmission channels. The dashed line is direct transmission channel from the source directly to the destination, while the combined solid lines are relay transmission channels from the source through the relay to the destination. The communication is divided into two orthogonal time slots in order to support the cooperation. During 
the first time slot, the source broadcasts its frame that contains data for the two destinations, and each destination receives its signal. In the second time slot, the source does not transmit and relay decodes and forwards the data flow to destination. Therefore, the destination node receives multiple copies of the same packets transmitted through different wireless channels, thus some degree of diversity can be obtained from such cooperative transmission strategies. It is worth noting that such cooperative communication brings significant improvement of reception reliability, which becomes an important criterion to measure the performance of cooperative transmissions and will be examined in following sections.

\section{A. Outage Behavior of Cooperative Transmission}

We employ a propagation model to consider path loss, shadow fading and Rayleigh fading. The wireless link $a_{i j}$ between the nodes $i$ and $j$ is modelled as $\mathrm{a}_{i j}=h_{i j} / d_{i j}^{k / 2}$, where $d_{i j}$, the distance between the nodes $i$ and $j$, represents the large-scale behavior of the channel gain, $k$ is the path-loss exponent and $h_{i j}$ captures the channel fading characteristics due to the rich scattering environment. In addition, the channel fading parameter $h_{i j}$ is assumed to be independent and identically distributed (i.i.d), complex Gaussian variable with zero mean and unit variance.

For direct transmission, the mutual information between a source and a destination is

$$
I_{D}=\log \left(1+\rho\left|\mathrm{a}_{s, d}\right|^{2}\right),
$$

where $\rho=E_{b} / N_{0}$ is defined as transmission power to noise ratio. Since for Rayleigh fading, $\left|\mathrm{a}_{s, d}\right|^{2}$ is exponentially distributed with parameter $d_{s, d}^{k}$. Thus, the outage probability satisfies

$$
\begin{aligned}
P_{D}^{\text {out }}=\operatorname{Pr}\left[I_{D}<R\right] & =1-\exp \left(-\frac{\left(2^{R}-1\right) d_{s, d}^{k}}{\rho}\right) \\
& =d_{s, d}^{k}\left(\frac{2^{R}-1}{\rho}\right) .
\end{aligned}
$$

where $R$ is the desired data rate in bit/s/Hz, which is defined by the quality of service (QoS) requirement and $d$ is the distance between two nodes.

For Cooperative transmission in Figure 1, let $d_{s, d}, d_{s, r}$ and $d_{r, d}$ be the respective distances among the source, relay and destination. During the first time slot, the destination receives $y_{d}=\frac{h_{s, d}}{d_{s, d}^{k / 2}} x_{s}+n_{d}$ from the source node, where $x_{s}$ is the information transmitted by the source and $n_{d}$ is white noise. During the second time slot, the destination node receives

$$
y_{d}= \begin{cases}\frac{h_{s, d}}{d_{s, d}^{k / 2}} x_{s}+n_{d}, & \text { if }\left|\frac{h_{s, r}}{d_{s, r}^{k / 2}}\right|^{2}<q\left(\rho_{s}\right) \\ \frac{h_{r, d}}{d_{r, d}^{k / 2}} x_{r}+n_{d}, & \text { if }\left|\frac{h_{s, r}}{d_{s, r}^{k / 2}}\right|^{2} \geq q\left(\rho_{s}\right)\end{cases}
$$

where $q\left(\rho_{s}\right)=\left(2^{2 R}-1\right) / \rho_{s}$ can be derived from direct transmission and is analogous to (2). As can be seen from the first condition of (3), when the link between the source and the relay is so poor that the relay is not able to decode, there is no performance gain can be achieved and the source is repeating its transmission during this slot. The second condition corresponds to the case when the relay can decode and repeat the source transmission, thus obtaining the secondorder diversity gain through CL. Therefore, choosing a proper relay to guarantee the link quality is critical in achieving good system performance.

Consider that a relay node is randomly selected. Hence the mutual information of this cooperative link can be shown as

$$
I_{C}= \begin{cases}\frac{1}{2} \log \left(1+2 \rho_{s}\left|\mathrm{a}_{s, d}\right|^{2}\right), & \left|\mathrm{a}_{s, r}\right|^{2}<q\left(\rho_{s}\right) \\ \frac{1}{2} \log \left(1+\rho_{s}\left|\mathrm{a}_{s, d}\right|^{2}+\rho_{r}\left|\mathrm{a}_{r, d}\right|^{2}\right), & \left|\mathrm{a}_{s, r}\right|^{2}>q\left(\rho_{s}\right)\end{cases}
$$

Therefore, the outage event for Minimum Power Selected Decode-and-Forward (MPSDF) is given by $I_{C}<R$ and is equivalent to the event

$$
\begin{aligned}
& \left(\left\{\left|\mathrm{a}_{s, r}\right|^{2}<q\left(\rho_{s}\right)\right\} \cap\left\{2\left|\mathrm{a}_{s, d}\right|^{2}<q\left(\rho_{s}\right)\right\}\right) \\
& \cup\left(\left\{\left|\mathrm{a}_{s, r}\right|^{2} \geq q\left(\rho_{s}\right)\right\} \cap\left\{\left|\mathrm{a}_{s, d}\right|^{2}+\left|\sqrt{\frac{\rho_{r}}{\rho_{s}}} \mathrm{a}_{r, d}\right|^{2}<q\left(\rho_{s}\right)\right\}\right),
\end{aligned}
$$

As we can see above, two events of the union in (5) corresponds to two cases in (4), respectively. Because the events in union of (5) are mutually exclusive, the outage probability becomes a sum

$$
\begin{aligned}
& P_{C}^{\text {out }}=\operatorname{Pr}\left[I_{C}<R\right] \\
& =\operatorname{Pr}\left[\left|\mathrm{a}_{s, r}\right|^{2}<q\left(\rho_{s}\right)\right] \operatorname{Pr}\left[2\left|\mathrm{a}_{s, d}\right|^{2}<q\left(\rho_{s}\right)\right] \\
& +\operatorname{Pr}\left[\left|\mathrm{a}_{s, r}\right|^{2} \geq q\left(\rho_{s}\right)\right] \operatorname{Pr}\left[\left|\mathrm{a}_{s, d}\right|^{2}+\left|\sqrt{\frac{\rho_{r}}{\rho_{s}}} \mathrm{a}_{r, d}\right|^{2}<q\left(\rho_{s}\right)\right] .(6)
\end{aligned}
$$

Here, we obtain a closed form expression for (5). By computing the limit, the large $\rho$ behaviour of (6) is (Let $\left.q\left(\rho_{s}\right)=q\right)$

$$
\begin{aligned}
& \frac{1}{q^{2}} P_{C}^{\text {out }}=\underbrace{\frac{1}{q} \operatorname{Pr}\left[\left|\mathrm{a}_{s, r}\right|^{2}<q\right]}_{1} \underbrace{\frac{1}{q} \operatorname{Pr}\left[2\left|\mathrm{a}_{s, d}\right|^{2}<q\right]}_{2} \\
& +\underbrace{\operatorname{Pr}\left[\left|\mathrm{a}_{s, r}\right|^{2} \geq q\right]}_{3} \underbrace{\frac{1}{q^{2}} \operatorname{Pr}\left[\left|\mathrm{a}_{s, d}\right|^{2}+\left|\sqrt{\frac{\rho_{r}}{\rho_{s}}} \mathrm{a}_{r, d}\right|^{2}<q\right]}_{4},
\end{aligned}
$$

where $1 \longrightarrow d_{s, r}^{k}, 2 \longrightarrow d_{s, d}^{k} / 2,3 \longrightarrow 1,4 \longrightarrow \frac{\rho_{s}}{\rho_{r}} d_{s, d}^{k} d_{r, d}^{k} / 2$. Then, above equation equals

$$
\frac{1}{q^{2}\left(\rho_{s}\right)} P_{C}^{\text {out }}=\frac{d_{s, d}^{k}}{2}\left(d_{s, r}^{k}+\frac{\rho_{s}}{\rho_{d}} d_{r, d}^{k}\right)
$$

Because $q\left(\rho_{s}\right)=\left(2^{2 R}-1\right) / \rho_{s}$, we have the outage probability between the source and the destination is

$$
P_{C}^{o u t}=\frac{1}{2} d_{s, d}^{k}\left(d_{s, r}^{k}+\frac{\rho_{s}}{\rho_{r}} d_{r, d}^{k}\right) \frac{\left(2^{2 R}-1\right)^{2}}{\rho_{s}^{2}} .
$$

where $\rho_{s}$ is the transmission power to noise ratio for the source node and $\rho_{r}$ is the transmission power to noise ratio for the relay node. 
TABLE I

POWER EFFICIENT Routing ALgORITHM

Initialize: Select the best possible relay node and establish a one-hop cooperative route (link) from the source to the destination to minimize the link BER of the route. Calculate the link BER according to (9) and compare it with the target BER (constraint).

Repeat: If any link BER along the constructed route is larger than the target BER, select new relay nodes for both relay links of the poor $\mathrm{CL}$ to improve its BER performance. Re-compute the link BER for the new constructed CLs and if the performance is satisfied the target, adjust the transmission power to minimal.

Stop: If all the link BER is equal or smaller than the target BER, then the cooperative route is finalized. Otherwise, continue with the repeat step.

\section{B. Power Minimization of Cooperative Link}

The signal-to-noise ratio (SNR) is defined as $\rho=E_{b} / N_{0}$ where $E_{b}$ denotes transmission energy per-bit and $N_{0}$ is the one-sided power spectral density of the white noise. Therefore, the transmission power can be expressed as $P=\rho N_{0} R B$. Assuming the assigned bandwidth is same for both source and relay nodes, outage probability (9) in terms of data rate, distances and transmission power can be further expressed as

$$
P_{C}^{\text {out }}=\frac{1}{2} d_{s, d}^{k}\left(d_{s, r}^{k}+\frac{P_{s}}{P_{r}} d_{r, d}^{k}\right) \frac{\left(2^{2 R}-1\right)^{2}\left(N_{0} R B\right)^{2}}{P_{s}^{2}} .
$$

where $P_{s}$ is the transmission power for the source and $P_{r}$ is the transmission power for the relay, $B$ is the bandwidth.

The optimization problem to minimize the total transmission power consumption of cooperative link with a target end-toend BER can be formulated as

$$
\begin{array}{ll}
\text { Minimize } & P_{s}+P_{r} \\
\text { Subject to. } & P_{C}^{\text {out }}<\mathrm{C}(\mathrm{C} \text { is the target BER })
\end{array}
$$

Using the Kuhn-Tucker condition, the minimum transmission power for MPSDF is shown as

$$
P_{s}=\frac{N_{0} R B}{d_{r, d}^{k}} \sqrt{\frac{m+1}{K}}, \quad P_{r}=\frac{N_{0} R B}{d_{s, r}^{k} m} \sqrt{\frac{m+1}{K}} .
$$

where $m=\frac{r+\sqrt{r^{2}+8 r}}{2}, r=\frac{d_{r, d}^{k}}{d_{s, r}^{k}}, K=\frac{2 \mathrm{C}}{d_{s, d}^{k} d_{s, r}^{k} d_{r, d}^{2 k}\left(2^{2 R}-1\right)^{2}}$.

Proof: See Appendix A.

\section{Description of the Power EfFicient Routing ALGORITHM}

Based on the characteristics of cooperative transmission analyzed at Section II, we propose here a distributed routing algorithm to establish a cooperative route in an arbitrary network that ensures each link BER below a certain target level (constraint). Table I describes the power efficient routing algorithm in detail. Each node uses a default transmission power to construct a route, in order to avoid high power consumption for long distance transmission at the initial stage. Each cooperative link can adjust to its minimum power using MPSDF once link performance is satisfied the target.

As a distributed routing algorithm, each relay node as a monitor periodically broadcasts a HELLO packet to its sourcedestination pair to measure the link performance. When an improvement is necessary, the relay sends a NOTIFICATION to its source and destination and triggers new relay selections between the source-relay and the relay-destination links. To fit the non-infrastructure nature of ad-hoc networks, it is desirable to devise a distributed mechanism to choose the relay node with the best incoming and outgoing channel condition among candidate nodes without using a central controller. In the proposed algorithm, relays use similar carrier sensing scheme [10] and go through a backoff period before sending received data to the destination. According to (11), since the minimum transmission power for both source and relay nodes can be determined by $d_{s, d}, d_{s, r}, d_{r, d}$ and link BER, relays can actually estimate these two power in the meantime of the relay node selection. Therefore, we propose a new relay selection scheme as following

1. In the first time slot, the source node broadcasts a test packet to the rests of nodes, each candidate relay node can estimate its $d_{s, r}$ and incoming channel condition through the received SNR strength. Meanwhile, the destination node can estimate $d_{s, d}$.

2. In the second time slot, the destination node broadcasts another test packet with information $d_{s, d}$, each candidate relay node can estimate $d_{r, d}$ and outgoing channel condition and extract $d_{s, d}$ from test packet.

For each candidate relay node, it can measure the BER performance and minimum power and set a back-off time which is proportional to the BER. The back-off period for each relay is chosen such that the smaller the link BER, the shorter the back-off time is. After the first back-off time expired, the corresponding relay node will broadcast an acknowledgment to other nodes which will quit the competition and refrain for next competition. At the same time, if the link BER is satisfied the constraint, a power adjustment will also be sent through the same acknowledgement to the source that both source and relay can adjust their power to optimal without adversely affecting the link performance. As a result, the selection of back-off periods at various relay nodes ensures that the best quality relay with optimized power will be the one responsible for forwarding the data to the destination node.

\section{NumERICAL RESUlTS}

In this section, we develop analytical results to illustrate the power savings of our proposed MPSDF transmission strategy and the power efficient routing algorithm, and then compare them with other cooperation-based power saving schemes.

\section{A. Power Reduction for MPSDF}

In this typical scenario, we assume the distance between one source and one destination is 100 meters. One single relay node is randomly placed in a $100 \mathrm{~m} \times 100 \mathrm{~m}$ area between the source and destination to form a cooperative link. Under the same path loss exponent $k=3, R=1 \mathrm{bit} / \mathrm{s} / \mathrm{Hz}$ and end-toend BER of 0.05 , we take 50 random experiments and the corresponding results are shown in Figure 2.

Due to performance enhancements, our proposed MPSDF always achieves much better energy performance than MPCR 


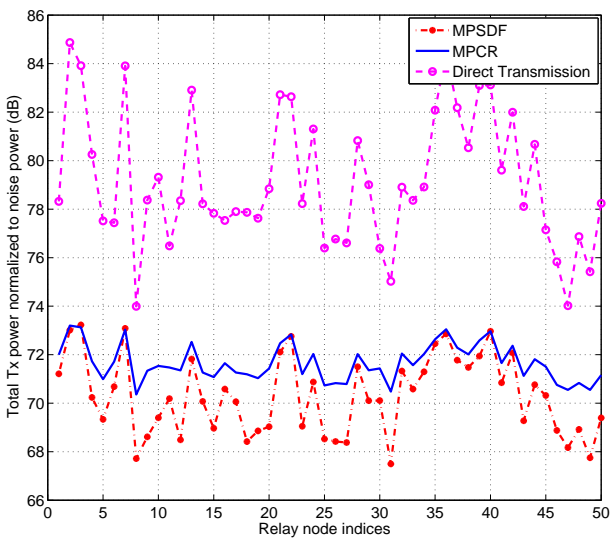

Fig. 2. Total normalized Tx power associated with relays at random locations

[9] as well as direct transmission, which goes through the same source-relay-destination links without cooperation. In addition, it is worth noting that total transmission power of proposed MPSDF is highly related with relay position, we can observe that for some points (e.g., node No. 31) of proposed scheme, total transmission power is relatively lower than other points, which is when the relay node nears to the destination node. That is so because the expression of total power (11) has the factors $m$ and $1 / K$, which is quite small when the relay node nears to the destination node. However, after 1000 simulation runs, the average power reduction for the MPSDF achieves $82.43 \%$ and $21.22 \%$, compared to direct transmission and MPCR, respectively.

According to the analysis above, it is clear that better located relay can further reduce power consumption. Therefore, using the same network topology in Figure 2, Figure 3 reports the minimum power consumption of cooperative link with the best located relay under same BER performance from four schemes. Results in Figure 3 are averaged over 1000 simulation runs. It is clearly seen that the best located relay with MPSDF achieves much better energy performance than the rest three schemes. In particular, when the BER constraint decreases, the total power consumption of MPCR closes to MPSDF. However, as we know from [9] that when BER is small, the MPCR acts as a direct transmission from the source directly to the destination with high probability. Whereas for MPSDF, both the source and the relay involves in cooperation. Hence the network lifetime of MPCR will be shorter due to high power consumption of the single source transmission.

\section{B. Power Comparisons between Routing Algorithms}

We consider here a network scenario that a total number of $\mathrm{N}$ nodes are uniformly distributed in a $1000 \mathrm{~m} \times 1000 \mathrm{~m}$ topology with the source and destination nodes located at the top left corner and the bottom right corner, respectively. Figure 4 shows the required total transmission power along the route with same total number of hops by different routing algorithms for different total number of nodes at same Link BER $=0.05$, $k=2$ and throughput $=0.95 \mathrm{bit} / \mathrm{s} / \mathrm{Hz}$, which is defined in

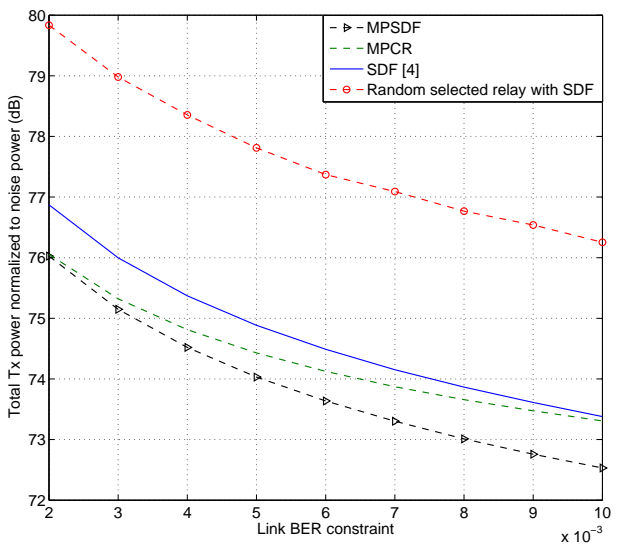

Fig. 3. Link BER vs. Total normalized Tx power consumption

[9]. As shown, the total power consumption decreases as the network size increases. This is so because the distance between neighbor nodes is reduced with increased node density. Multihops routing ensures the lower power consumption between these nodes. We can also observe that the proposed power efficient routing algorithm achieves better performance than CASNCP [9] which is based on the shortest path algorithm, the centralized algorithm [11] and DSDV algorithm [12].

Since our proposed routing algorithm starts with routes with a small number of hops, Figure 5 shows the relationship between the total power consumption of cooperative route in terms of total number of hops and its end-to-end BER performance.

The network scenario is same as in Figure 4, but with a fixed $\mathrm{N}=100, k=2$ and $R=0.2 \mathrm{bit} / \mathrm{s} / \mathrm{Hz}$. Since the total transmission power along the path is proportional to total number of hops. Under the same network topology, as the total number of hops increases, the total transmission power is increased. Meanwhile, under the same route and end-to-end BER achievement, the proposed algorithm can reduce the total power consumption by a couple dB.

\section{CONCLUSIONS}

In this paper, we have investigated the performance benefits of cooperative communication on power reduction in wireless networks. For a given source-destination pair, a new distributed cooperative transmission strategy MPSDF has been first proposed for realizing minimum power transmission. The key contribution of the proposed scheme is to bring the performance gain of cooperative diversity from the physical layer up to the networking layer. Analytical results have shown that MPSDF achieves average energy saving of $82.43 \%$ compared to direct transmission, and of $21.22 \%$ compared to MPCR. Furthermore, we have proposed the distributed and power efficient cooperative routing algorithm, which constructs the minimum power route with a small number of hops. From the simulation results, the total power consumption of our proposed routing algorithm can reduce by a couple $\mathrm{dB}$ compared to the existing cooperative routing algorithms. 


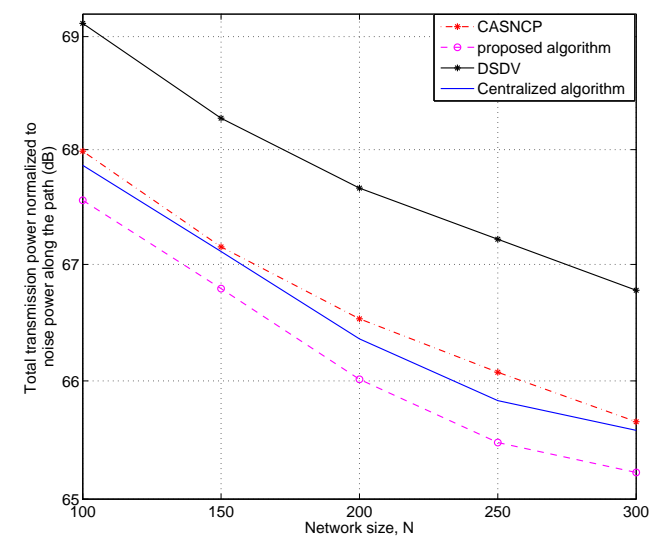

Fig. 4. Network size vs. Total normalized Tx power consumption along the route

\section{APPENDIX A}

According to the Kuhn-Tucker condition, the inequality constraints can be converted to the equality constraints. We set $k=2, \rho_{s}=\rho_{1}, \rho_{r}=\rho_{2}$ Then, we have

$$
\begin{aligned}
& P_{C}^{\text {out }}=\frac{1}{2} d_{s, d}^{2}\left(d_{s, r}^{2}+\frac{\rho_{1}}{\rho_{2}} d_{r, d}^{2}\right) \frac{\left(2^{2 R}-1\right)^{2}}{\rho_{1}^{2}}=C \\
& \Longrightarrow 1+\frac{d_{r, d}^{2} \rho_{1}}{d_{s, r}^{2} \rho_{2}}=\frac{2 C \rho_{1}^{2}}{d_{s, d}^{2}\left(2^{2 R}-1\right)^{2} d_{s, r}^{2}}
\end{aligned}
$$

We let $d_{r, d}^{2} \rho_{1}=\widetilde{\rho_{1}}, d_{s, r}^{2} \rho_{2}=\tilde{\rho_{2}}$, then we can have the following $1+\frac{\widetilde{\rho_{1}}}{\widetilde{\rho_{2}}}=\frac{2 C{\widetilde{\rho_{1}}}^{2}}{d_{s, d}^{2}\left(2^{2 R}-1\right)^{2} d_{s, r}^{2} d_{r, d}^{4}}$.

We also assume $\frac{2 C{\widetilde{\rho_{1}}}^{2}{ }^{2, r}{ }^{2}{ }_{s, d}\left(2^{2 R}-1\right)^{2} d_{s, r}^{2} d_{r, d}^{4}}{{ }_{c}}=K$. Finally, the optimization problem can be simplified as

$$
\begin{array}{ll}
\text { Minimize } & \frac{\widetilde{\rho_{1}}}{d_{r, d}^{2}}+\frac{\widetilde{\rho_{2}}}{d_{s, r}^{2}} \\
\text { Subject to. } & 1+\frac{\widetilde{\rho_{1}}}{\rho_{2}}=K{\widetilde{\rho_{1}}}^{2}
\end{array}
$$

We assume $K{\widetilde{\rho_{1}}}^{2}-1=\tan ^{2} x=\sec ^{2} x-1$. Then

$$
\begin{gathered}
\sqrt{K} \widetilde{\rho_{1}}=\sec x \Longrightarrow \widetilde{\rho_{1}}=\frac{\sec x}{\sqrt{K}} . \\
\frac{\widetilde{\rho_{1}}}{\widetilde{\rho_{2}}}=\tan ^{2} x \Longrightarrow \widetilde{\rho_{2}}=\frac{\widetilde{\rho_{1}}}{\tan ^{2} x}=\frac{\cos x}{\sqrt{K} \sin ^{2} x} .
\end{gathered}
$$

After that, we get $\frac{\widetilde{\rho_{1}}}{d_{r, d}^{2}}+\frac{\widetilde{\rho_{2}}}{d_{s, r}^{2}}=\frac{1}{d_{r, d}^{2} \sqrt{K} \cos x}+\frac{\cos x}{d_{s, r}^{2} \sqrt{K} \sin ^{2} x}$.

Up to this step, we transform two variables into one variable. If $\rho_{1}+\rho_{2}$ can have the minimal value, then the first order of $\rho_{1}+\rho_{2}$ should be $\frac{d\left(\rho_{1}+\rho_{2}\right)}{d x}=0$. Because $\frac{1}{\sqrt{K}}$ is a constant, the first order of $\rho_{1}+\rho_{2}$ is equal to

$$
\begin{aligned}
& \frac{d\left(\rho_{1}+\rho_{2}\right)}{d x}=\frac{1}{d_{r, d}^{2}}(-1) \cos ^{-2} x(-\sin x) \\
& +\frac{1}{d_{s, r}^{2}}\left[(-\sin x) \sin ^{-2} x+\cos x(-2) \sin ^{-3} x \cos x\right]=0 \\
& \Longrightarrow \frac{\sin x}{d_{r, d}^{2} \cos ^{2} x}=\frac{1}{d_{s, r}^{2}}\left[\frac{1}{\sin x}+2 \frac{\cos ^{2} x}{\sin ^{3} x}\right] \\
& \Longrightarrow \tan ^{2} x=\frac{d_{r, d}^{2}}{d_{s, r}^{2}}+2 \frac{d_{r, d}^{2}}{d_{s, r}^{2}} \tan ^{-2} x
\end{aligned}
$$

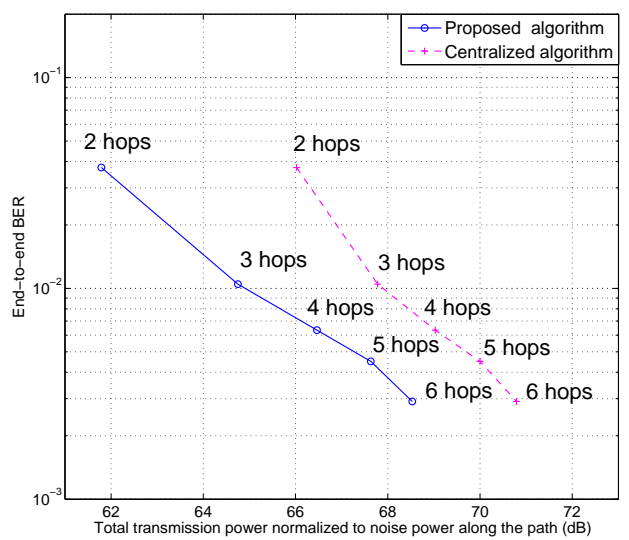

Fig. 5. Total normalized Tx power along the path vs. End-to-end BER

Define $\tan ^{2} x=m$, (15) equals to $m-\frac{d_{r, d}^{2}}{d_{s, r}^{2}}-2 \frac{d_{r, d}^{2}}{d_{s, r}^{2} m}=0$. Further assuming $r=\frac{d_{r, d}^{2}}{d_{s, r}^{2}}$, we have $m^{2}-r m-2 r=0$. Then the roots are $m=\frac{r \pm \sqrt{r^{2}+8 r}}{2}$. Since $\tan ^{2} x>0, m=$ $\frac{r+\sqrt{r^{2}+8 r}}{2}$.

Finally, the optimized SNR ratios for source and relay are $\rho_{1}=\frac{\widetilde{\rho_{1}}}{d_{r, d}^{2}}=\frac{1}{d_{r, d}^{2}} \sqrt{\frac{m+1}{K}}, \quad \rho_{2}=\frac{\widetilde{\rho_{2}}}{d_{s, r}^{2}}=\frac{1}{d_{s, r}^{2} m} \sqrt{\frac{m+1}{K}}$.

Consider the power $P=\rho N_{0} R B$ and $\hat{d}=d^{k / 2}$, we get the final result.

\section{REFERENCES}

[1] J. H. Chang and L. Tassiulas, "Maximum lifetime routing in wireless sensor networks," IEEE/ACM Transactions on Networking, vol. 12, August 2004.

[2] C. K. Toh, "Maximum battery life routing to support ubiquitous mobile computing in wireless ad hoc networks," IEEE Communications Magazine, 2001.

[3] A. Scaglione, D. Goeckel, and J. N. Laneman, "Cooperative communications in mobile ad-hoc networks: Rethinking the link abstraction," IEEE Signal Processing Magazine, vol. 23, pp. 18-29.

[4] J. N. Laneman, D. N. Tse, and G. W. Wornell, "Cooperative diversity in wireless networks: Efficient protocols and outage behavior," IEEE Trans. On Information Theory, vol. 50(12), Dec. 2004.

[5] A. E. Khandani, "Cooperative routing in wireless networks," Ph.D. dissertation, MIT, 2004.

[6] K. W. F. Li and A. Lippman, "Energy-efficient cooperative routing in multi-hop wireless ad hoc networks," in IEEE IPCCC, 2006.

[7] C. Pandana, W. P. Siriwongpairat, T. Himsoon, and K. J. R. Liu, "Distributed cooperative routing algorithm for maximizing network lifetime," in IEEE Wireless Communications and Networking Conference, 2006.

[8] W. Ge, J. Zhang, and G. Xue, "Joint clustering and optimal cooperative routing in wireless sensor networks," in IEEE International Conference on Communication, ICC, 2008.

[9] A. S. Ibrahim, Z. Han, and K. J. R. Liu, "Distributed energy- efficient cooperative routing in wireless networks," in IEEE Global Telecommunication Conference, Globecom, 2007.

[10] S. Zhu and K. K. Leung, "Jdistributed cooperative routing for uwb adhoc networks," in IEEE International Conference on Communication, ICC, 2007.

[11] Z. Sheng, Z. Ding, and K. K. Leung, "On the design of a quality-ofservice driven routing protocol for wireless cooperative networks," in IEEE Vehicular Technology Conference, Singapore, 2008.

[12] C. E. Perkins and P. Bhagwat, "Highly dynamic destination c sequenced distance-vector routing (dsdv) for mobile computers," in Proc of ACM SIGCOMM, 1994 